\title{
Comentarios sobre el problema de empleadores e inmigración de indocumentados a EUA: una oportunidad para estudios macro regionales
}

\author{
Joseph Nalven \\ Instituto de Estudios Regionales de las Californias \\ Universidad de San Diego
}

\begin{abstract}
Resumen
El fenómeno de trabajadores de origen mexicano en Estados Unidos tiene impactos nacionales y transnacionales en México y en aquel país. Existen contrastes importantes: unos señalan la contribución económica que hacen los inmigrantes mexicanos a ambos países mientras mejoran su vida propia; otras señalan la competencia en el mercado laboral en los Estados Unidos, la cual empeoró la situación de los trabajadores nacionales de Estados Unidos. No es el propósito resolver estos dos puntos de vista, sino revisar algunos estudios sobre empleadores, puesto que la legislación implica un mayor control de empleadores con sanciones en los sectores urbanos, mientras que hace excepciones extraordinarias a los agricultores. El resultado de este trabajo nos ofrece solo una visión parcial e incompleta de cómo varía entre empresas grandes y pequeñas el empleo de inmigrantes - legales o indocumentados - en varios sectores de la economía y sobre todo, la variación microregional.
\end{abstract}

Palabras clave: mercado laboral, empleadores norteamericanos, migración indocumentada, estudios micro-regionales.

\begin{abstract}
The phenomenon of Mexican workers in the United States has national and trans-national impact in Mexico and in that country. There are important contrasts: The first one highlights the economical contribution that Mexican immigrants give to the two countries while they improve their levels of life. The other one is focused on the competitiveness of labor market in the United States, which negatively affected native workers of the United States. It is not the objective of this essay to solve these two points of view, but to review some researches performed on employers, since new regulations put more control to employers by sanctioning those who are in urban areas; while making extraordinary exemptions to farmers. The result of this project is only a partial an incomplete vision on how different the circumstances of immigrant employment are, between small and large companies - either they are legal or undocumented -according to the diverse economical sectors, and mainly to the microregional variations.
\end{abstract}

Keywords: labor market, American employers, undocumented migration, micro-regional researches. 


\title{
COMENTARIOS SOBRE EL PROBLEMA DE EMPLEADORES E INMIGRACION DE INDOCUMENTADOS A EUA: UNA OPORTUNIDAD PARA ESTUDIOS MACRORREGIONALES
}

\author{
Por
Joseph Nalven, Ph. D.*
}

Nuevamente los debates sobre inmigración se repiten en el congreso de Estados Unidos. Dos elementos en la legislación tienen interés para los estudiantes del mercado laboral: sanciones contra empleadores y un nuevo programa de braceros o "trabajadores huésped". Los estudiosos del mercado laboral por lo general han observado que el fenomeno de trabajadores de origen mexicano en E.U.A. tiene impactos nacionales y transnacionales en México y en aquel país. Hay un contraste importante: unos señalan la contribución económica que hacen los inmigrantes mexicanos a ambos países mientras mejoran su vida propia; otras señalan la competencia en el mercado laboral en los Estados Unidos (más indirecta que directa) en la que los estándares salariales y ocupacionales (como salud) se empeoraron para el trabajador nacional de E.U.A.

El fin de esta ponencia no es resolver o in tegrar estos dos puntos de vista sino más bien, revisar algunos estudios sobre empleadores, puesto que la legislación implica un mayor control de empleadores con sanciones en los sectores urbanos, mientras que hace excepciones extraordinarias a los agricultores.

El 15 de noviembre de 1985, el Comité de Relaciones Intergubernamentales de la Comisión de las Californias tuvo una junta sobre el tema de inmigración indocumentada y sus impactos sobre las relaciones California/México. El comité buscaba discutir las "implicaciones del tema". Los conceptos que presenté en esa reunión fueron sobre el futuro, en el caso de que tal legislación se implementara.

La recomendación fue sencilla: crear una Comisión Californiana sobre inmigración para hacer un monitoreo continuo del impacto en el estado de California. Es obvio que tal propuesta no resuelve la división palpable entre las varias funciones del debate sobre inmigración, pero sí puede enfocar la conciencia californiana sobre impactos fiscales y sociales frente a la opinión nacional. Tal vez hay otras alternativas. Un ejemplo es la decisión tomada por la Comisión de Relaciones de Trabajo Agrícola en octubre del presente año. La Comisión negó aceptar la decisión de la Suprema Corte de "Sure-Tan, Inc. v. NLRB_U.S._, $1{ }^{\circ} 4$ S.Ct. 2803 (1984)" en la que el empleador dio de baja

\footnotetext{
* Institu to para Estudios Regionales de las Californias Universidad de San Diego.
} 
a varios trabajadores y no tuvo que pagar la parte del salario correspondiente a horas extras puesto que los trabajadores eran indocumentados. La justificación de la Comisión de Relaciones de Trabajo Agrícola (Agricultural Relations Board) fue que la legislación californiana fue distinta a la ley federal y no estableció precedente legal (Rigi Agricultural Services, Inc. v. United Farm Workers of America, AFL-CIO, 11 ALRB No. 27, October 28, 1985). Entonces el factor de una conciencia californiana puede afectar la forma y resultados de legislación federal. Volviendo a la reunión del 15 de noviembre de 1985, del Comité de Relaciones Intergubernamentales de la Comisión de las Californias un experto sobre el mercado laboral de imigrantes, el Dr. Richard Mines, presento un resumen de sus estudios durante ocho años, terminando con su colaboración con El Centro de Estudios México-Estados Unidos (de la Universidad de California. San Diego). La conclusión que presentó fue:

"Creo que necesitamos inmigrantes, pues son un beneficio a nuestra sociedad; pero necesitamos inmigrantes que eventualmente lleguen a ser ciudadanos completos, no de tipo temporal--los que se pueden aprovechar fácilmente."1

"La inmigración por sí misma no es un problema. Comunidades inmigrantes en California han iniciado auges económicos que estimularon empleo para sí mismos y para trabajadores domésticos. El problema está en el funcionamiento de los mercados laborales. Trabajadores sin o con poca calificación, ocupando posiciones que tradicionalmente han sido sindicalizadas, han perdido una buena parte de sus habilidades para defenderse en el puesto de trabajo. Algunos empleadores estan dispuestos a aceptar una alta tasa de rotación con bajos salarios y a utilizar un sistema de competencia entre hispanos ("leadman system"), en que un grupo de inmigrantes se enfrenta competitivamente a otro grupo de inmigrantes; han aprovechado un clima político y social muy favorable a ello para degradar las condiciones de trabajo de los empleados". 2

El resultado, según Richard Mines, es que no existe muy poco desplazamiento directo de inmigrantes entrando por una puerta a trabajar y trabajadores nativos de E.U.A. saliendo por la otra sin trabajo. Pero sí hay un proceso de no emplear trabajadores estadounidenses por parte de los empleadores. Se da un ejemplo de 1,500 a 2,000 trabajadores de color en Los Angeles que fueron abandonados por empleadores. Los encargados de mantenimiento recibían $\$ 13$ dólares por hora y eran sindicalizados. Pero los empleadores cam-

${ }_{1}$ Richard Mines, Undocumented immigrants and California industries, reflections on research, for hearing on the Intergobemmental Relations Committee, Calfornia Assembly, Sacramento, November 15, 1985, p. 11 .

2Richard Mines, Employer's use of low-wage immigrants in California: a dilemma for liberal, unpublished paper, August 1985, p. 26-27. 
biaron contratos y de inmediato tuvieron acceso a las redes de inmigrantes recientes. El salario bajó a un poco más que el mínimo (el mínimo es de \$3.35 la hora); el sindicato perdió y la mayoría de los negros quedaron desocupados. 3

Hay beneficios y costos en el proceso de abandonar trabajadores domésticos: beneficios a los nuevos inmigrantes, a los empleadores y a los consumidores de este servicio; costos a los abandonados y al Estado, que paga el seguro de desempleo, estampillas para comida, asistencia social y seguros médi$\cos$.

Mines ha hecho claro que el proceso de abandonar trabajos no calificados no es nuevo, pero tiene una historia larga en E.U.A. que se domina "el cambio étnico lento" (slow ethnic shift). ${ }^{4}$ La clave es el empleador, que tie. ne la libertad de seleccionar a sus trabajadores. Algunos empleadores aprovechan el clima político y social para degradar las condiciones de trabajo de los indocumentados.

Otro investigador, Wayne Cornelius, empleó, al igual que Mines, datos de la misma muestra de empleadores en San Diego, Los Angeles, y San Francisco. Aquí se presentan algunas estadísticas que comparan las empresas grandes (número promedio de trabajadores $=300$ ) con las pequeñas (número promedio de trabajadores $=39$ ). En las pequeñas, los trabajadores están menos sindicalizados $(27.3 \%)$ que en las grandes $(54 \%)$ y les pagan menos ( $\$ 3.75$ y $\$ 4.12$ por hora, respectivamente). Es interesante hacer notar que ambos tienen un número promedio de $50 \%$ de trabajadores monolingües en español. Tal vez el hallazgo más importante es que tanto las compañías grandes (c.g.) como las pequeñas (c.p.) no tienen flexibilidad amplia para reclutar trabajadores nativos: solamente el $23.8 \%$ (c.p.) y el $36.5 \%$ (c.g.) no tuvieron cambios básicos en sus operaciones y pudieron emplear trabajadores substituidos por indocumentados (cuadro 1).

Estos resultados implican, para Cornelius, que si el gobierno federal implementa una nueva legislación que tiene sanciones contra empleadores, las compañías grandes darán de baja a sus indocumentados y al mismo tiempo, "subcontratarían más trabajo con las compañías pequeñas, no sindicalizadas, y que son dominadas por inmigrantes. Estas compañías pequeñas continuarán empleando a los indocumentados - ley o no ley-porque tienen que hacerlo para mantener su posición competitiva." 5

3Richard Mines, Undocumented im migrants ..., pp. 4-5.

4Richard Mines, "Employer's Use ...,", 15.

5Wayne A. Cornelius, "The role of Mexican labor in the U.S. Economy: two generations of research", (forthcoming in Los Angeles Business-Labor Council, Immigration and jobs in Los Angeles: Current impacts, future trends, proceedings, labor market information project, Los Angeles Business-Labor Council, February 15, 1985, p. 17). 


\section{CUADRO 1. Comparación entre grandes y pequeñas empresas sobre variables seleccionadas (muestra total).}

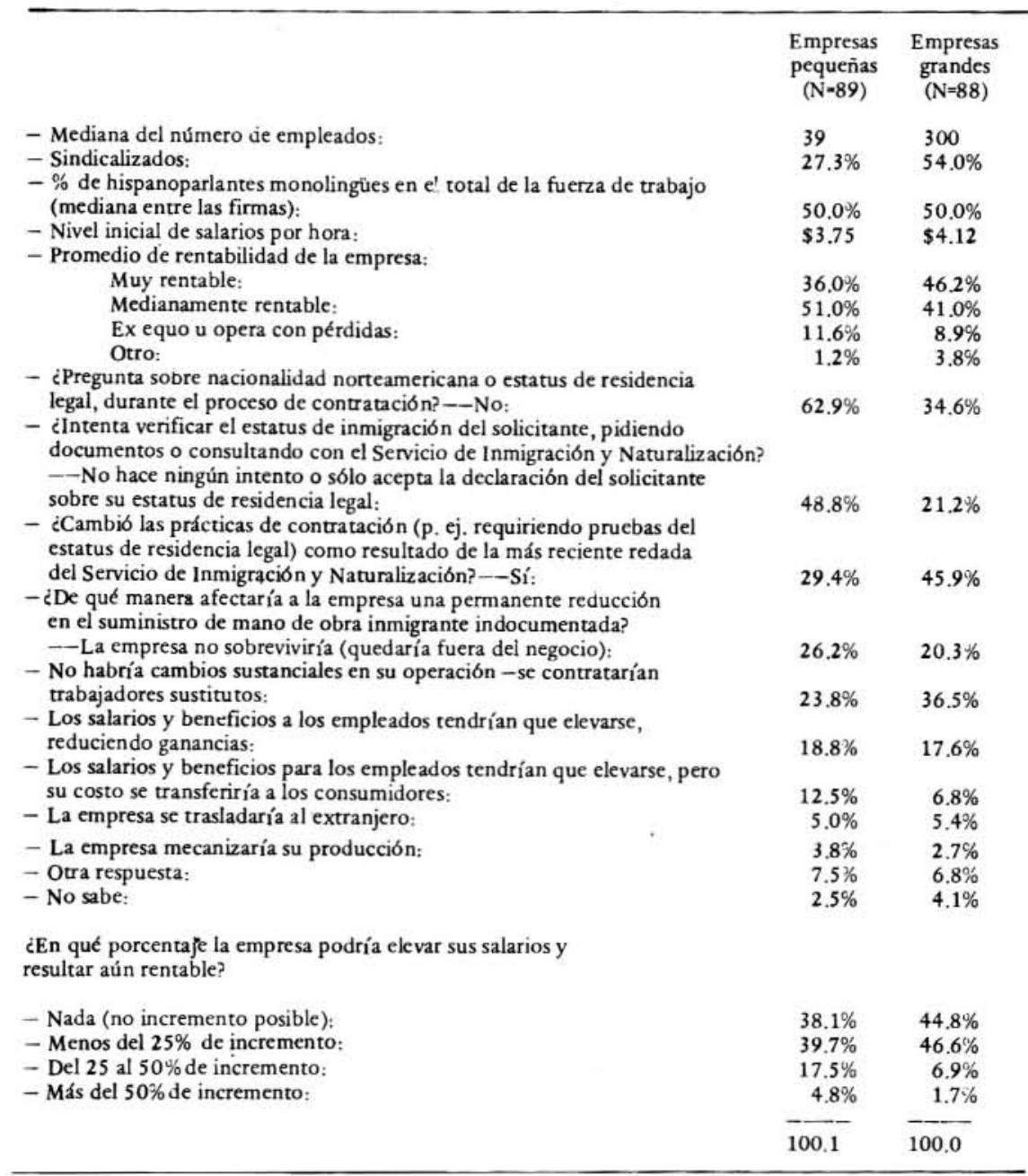

FUENTE: "El Papel del Trabajo Mexicano en la Economía de Estados Unidos: Dos Generaciones de Investigación", por Wayne Comelius, documento presentado en la Confrencia sobre Inmigración y Empleos en Los Angeles: Impactos actuales, futuras tendencias, Los Angeles, Proyecto de Informadión sobre Merrados de Trabajo, Los Angeles Business Labor Council, febrero 15 de 1985. 
Desafortunadamente, en las alternativas del cuestionario no se incluyó la pregunta de si las compañáas grandes usarían la táctica de subcontratar a las compañías pequeñas. Quizás el investigador no creía que le dieran una respuesta honesta, pero el mismo riesgo tuvo con todas las otras alternativas. No hay comentario en su reporte que explique la metodología y las razones por las que estableció tales alternativas. Así, tenemos una inferencia de comportamiento. Cornelius cree que la intervención federal empujará a los indocumentados hacia las compañías pequeñas, con una notoria presión hacia bajar los salarios, obstaculizando aún mas la sindicalización de la fuerza laboral. 6

La posición de Cornelius es en parte contraintuitiva, puesto que la posición oficial del sindicato más grande en E.U.A., AFL-CIO, es la de respaldar sanciones contra empleadores que usan indocumentados.

Segundo, y pensando en su compañero de campo Richard Mines, el proceso del subcontratador de abandonar los trabajadores de alto costo, sindicalizados, ya ha empezado; no se tiene que culpar a la nueva legislación ni a las prácticas del Servicio de Inmigración y Naturalización (INS). La fuerza natural del mercado da razón suficiente para explicar este proceso. ¿Por qué buscar a un Deus ex machina para explicar un proceso que ya tiene una explicación?

Quisiera dar un ejemplo de otro estudio hecho en San Diego, que es parte de la línea de estudios sobre indocumentados en San Diego y la primera que trata el tema de los empleadores. Este estudio, "La perspectiva de empleadores: sí existe la necesiaad de un programa de trabajadores huésped", da la opinión de que los agricultores culpan a los sindicatos por las alzas de salarios en el sur del condado. Pero tras un análisis de la historia del cultivo del tomate, se descubrió que antes de la firma del contrato con los sindicatos, los agricultores en el norte del condado quitaron a los "raiteros" (trabajadores legales que cruzan la frontera desde Tijuana todos los días) y los reemplazaron con indocumentados. Claro, los agricultores del norte aprovecharon la inhabilidad del INS para controlar el flujo migratorio.

¿A quién se le debe culpar? ¿A los sindicatos por tratar de mejorar la vida de sus afiliados? ¿Al INS por no sacar a los indocumentados del norte con la misma eficiencia como lo hace en el sur del condado? ¿O al empleador en el norte que pudo aprovechar la situación para ganar una ventaja económica? ¿O, como es la opinión popular, culpar a los trabajadores nativos por no competir con el indocumentado?

Cualquier respuesta que vea la competencia del mercado laboral solamente de un lado de la frontera no puede ser completa. Indudablemente se 6Ibid., p. 18. 
necesita un acuerdo, formal o informal, que responda a la dinámica asimétrica en ambos lados de la frontera. En un nivel de entendimiento, se conoce la participación mexicana en el mercado laboral de los E.U.A.; pero, en otro nivel, nos falta una visión completa de la dinámica laboral, especialmente la variación entre empleadores en la selección de trabajadores por características de experiencia, nivel de educación, sexo, raza, edad, estatus legal, etc. Por ejemplo, Cornelius dio énfasis al tamaño de la compañá, grandes versus pequeñas. Sí, este es un factor de importancia. Pero se tiene la misma influencia en varios sectores que tienen sistemas distintos de organización, factores de ubicación diferentes, factores de imagen distintos en relación con la comunidad y entre regiones geográficas. Este es por lo tanto sólo un factor de interés frente a un fenómeno bastante complejo.

Comparemos ahora el estudio del Centro de Estudios de Estados Unidos y México, en el cual estuvieron afiliados Wayne Cornelius y Richard Mines, con otro estudio elaborado en San Diego por Community Research Associates en 1981. En este último, en el cual me tocó ser el investigador principal, hay un análisis de dos etapas acerca de la variación de tres sectores de la economía -agricultura, servicios, y manufacturas-, representados por granjas agrícolas, restaurantes y electrónica.

En la primer etapa del análisis, encontramos el hecho de que los empleadores que tienen un empleo extenso de indocumentados, o que son dependientes del indocumentado, vaticinan el cierre de su negocio con más frecuencia que los empleadores que tienen un empleo limitado de indocumentados, $90 \%$ a $10 \%$ (cuadro 2). Esto es un resultado obvio cuando se toma en cuenta al alza de salario que resultara si se tuviera que atraer al trabajador nacional (de E.U.A.).

La segunda etapa del análisis estu dia la muestra de empleadores, no como empresas con ocupación extensa o limitada de indocumentados, sino como empresas con sus propios problemas, dinámica y oportunidades. La reacción de los empleadores a la contratación de indocumentados versus nacionales tiene un patrón distinto que varía según la ideología de cada in dustria. Se ven respuestas completamente distintas en estos tres sectores (cuadro 3 ). Los agricultores, que representan una actividad que tiene un uso extenso de indocumentados, generalmente reflejan la actitud de que los trabajadores indocumentados representan un costo más bajo que los trabajadores nacionales $(86 \%)$, trabajan con más productividad (86\%), aumentan la productividad ( $86 \%)$, no son menos confiables que los trabajadores nacionales (64\%), $y$ dudan de que puedan sustituir a los trabajadores indocumentados por trabajadores nacionales. Los agricultores están en desacuerdo con la idea de sancionar a los empleadores. 
CUADRO 2. Respuestas de empleadores al alza de $25 \%$ por trabajo "X".

\begin{tabular}{|c|c|c|c|c|c|}
\hline \multirow{2}{*}{ Uso } & \multirow[t]{2}{*}{$\begin{array}{c}\text { CIERRE DEL } \\
\text { NEGOCIO }\end{array}$} & \multirow[t]{2}{*}{$\begin{array}{c}\text { OTRO } \\
\text { RESULTADO }\end{array}$} & \multirow[t]{2}{*}{$\begin{array}{c}\text { TERMINA EL } \\
\text { NEGOCIO }\end{array}$} & \multicolumn{2}{|c|}{$\begin{array}{c}\text { OTRO } \\
\text { RESULTADO }\end{array}$} \\
\hline & & & & & \\
\hline limitado & 1 & 22 & $\begin{array}{r}4 \% \\
10 \% \\
64 \%\end{array}$ & $\begin{array}{l}96 \% \\
84 \% \\
36 \%\end{array}$ & $\begin{array}{l}100 \% \\
100 \%\end{array}$ \\
\hline $\begin{array}{l}\text { Uso } \\
\text { extenso }\end{array}$ & 9 & 5 & $\begin{array}{r}90 \% \\
100 \%\end{array}$ & $\begin{array}{r}16 \% \\
100 \%\end{array}$ & \\
\hline \multicolumn{6}{|c|}{$\begin{array}{l}\text { Impacto pri } \\
\mathrm{N}=37 \\
\mathrm{P}=.00013\end{array}$} \\
\hline Uso de in & cumentados & & & & \\
\hline
\end{tabular}

FUENTE: The employer's view: is there a need for a Guest-Worker Program?, by Joseph Nalven and Craig Frederickson, San Diego: Community Research Associates, 1982, p. 59.

Los empleadores de electrónica en San Diego, donde el uso de indocumentados es limitado (pues cuenta con los refugiados asiáticos como trabajadores legales que tienen muchas de las características de los indocumentados, incluyendo su apatía por sindicalizarse y la aceptación de salarios bajos, no tienen tantas inquietudes sobre las condiciones del empleo, etc.,) presentan un patrón de actitudes opuestas a los agricultores. Estos reflejan la actitud de que la compañía no logra un costo laboral más bajo con el uso de indocumentados, que los indocumentados no trabajan con más productividad, no aumentan la productividad, son menos confiables, y pueden ser substituidos completamente con trabajadores nacionales. Los empleadores en electrónica favorecen el uso de sanciones contra empleadores que contratan trabajadores indocumentados. Lógicamente, los empleadores en restaurantes, que tienen un empleo de indocumentados ubicado entre los dos extremos (agricultores y manufactoreros), deben tener un patrón de actitudes entre los dos también; y en general, los resultados confirman esta predicción.

Hasta aquí he dado unos ejemplos sobre los empleadores en San Diego (y en el primer estudio, con una muestra que incluye Los Angeles y San Francisco). Como se decía anteriormente, se tiene una visión parcial e incompleta, especialmente en el mercado laboral transfronterizo, de cómo varía entre empresas grandes y pequeñas el empleo de inmigrantes -legales e 
indocumentados- en varios sectores de la economía, y sobre todo, la variación microrregional.

\section{CUADRO 3. Actitudes de empleadores comparando el indocumentado CUADRO 3. al trabajador nacional (U.S.A.).}

\section{PREGUNTA \\ 1. La compañía tendrá un costo laboral más bajo porque los ciudadanos no aceptarán trabajo por el salario preva-} PORCENTAJE: "DE ACUERDO" $O$ " $S$ "

\section{Agricultura}

Kestaurantes

Electrónica leciente.

2. Los indocumentados trabajan con mas productividad que los ciudadanos (U.S.A.)

$86 \%$

$56 \%$

$11 \%$

3. ¿El empleo de indocumentados aumenta la productividad de las compañías en su sector?

$86 \%$

$27 \%$

$8 \%$

4. ¿Los indocumentados son menos confiables que los ciudadanos (U.S.A.)?

$36 \%$

$11 \%$

$56 \%$

5. ¿Hasta qué punto puede sustituir trabajadores (U.S.A.) por indocumentados?

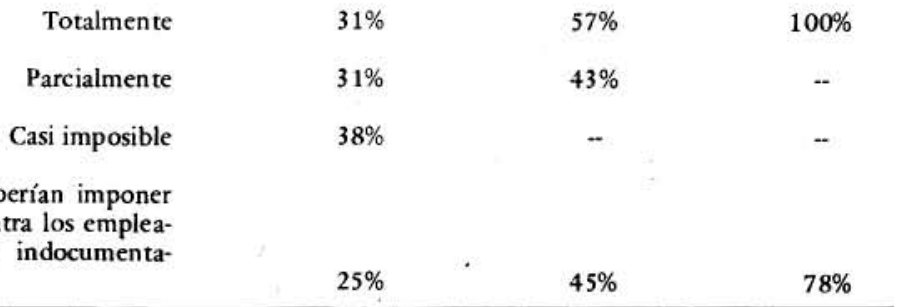

FUENTE: The employer's view: is there a need for a Guest-Worker Program? by Joseph Nalven and Craig Frederickson, San Diego: Community Research Associates. 1982, p. 59. 